\title{
On the dependence of the Bergman function on deformations of the Hartogs domain
}

\author{
by ZBigniew PAsternak-Winiarski (Warszawa)
}

\begin{abstract}
We apply the Rudin idea to represent the Bergman kernel of the Hartogs domain as the sum of a series of weighted Bergman functions in the study of the dependence of this kernel on deformations of the domain. We prove that the Bergman function depends smoothly on the function defining the Hartogs domain.
\end{abstract}

1. Introduction. The problem of the dependence of the Bergman function on deformations of the domain has been considered by Greene and Krantz in the papers [3] and [4]. In [4] it is proved that for any $\varrho>0$ the restriction $K_{D \mid \bar{D} \times \bar{D} \backslash \Delta_{e}}$ of the Bergman function $K_{D}$ of a $C^{\infty}$ strongly pseudoconvex domain $D$ in $\mathbb{C}^{n}$ to the set $\bar{D} \times \bar{D} \backslash \Delta_{\varrho}$, where $\Delta_{\varrho}:=\{(z, w) \in$ $\bar{D} \times \bar{D}:|z-w|+\operatorname{dis}(z, \partial D)+\operatorname{dis}(w, \partial D)<\varrho\}$, depends continuously on $D$ in the $C^{\infty}$ topology. In this paper we suppose that $D=\Omega(\varphi, m) \subset \mathbb{C}^{n+m}$ is a Hartogs domain defined by a fixed open bounded set $\Omega \subset \mathbb{C}^{n}$, a natural number $m$ and a lower semicontinuous positive bounded function $\varphi: \Omega \rightarrow \mathbb{R}$ (see Section 3). In Section 4 we show (see Theorem 2) that for any compact set $Z \subset \Omega(\varphi, m) \times \Omega(\varphi, m)$ the restriction $K_{\Omega(\varphi, m) \mid Z}$ depends smoothly on $\varphi$. Here we consider $K_{\Omega(\varphi, m) \mid Z}$ as an element of the Banach space $C(Z)$ of all continuous complex-valued functions on $Z$. In the proof of Theorem 2 we use the Rudin idea to represent the Bergman function on the Hartogs domain $\Omega(\varphi, m)$ as the sum of some series of weighted Bergman functions on $\Omega$ (see [2], [6], [7] or [13]). This approach allows us to make the most of the results on weighted Bergman functions obtained in [11]. The necessary definitions and facts concerned weighted Bergman functions are collected in Section 2. The properties of the Bergman function on a Hartogs domain as well as a suitable differentiable structure on the set $L S P(\Omega)$ of all lower semicontinuous positive bounded functions on $\Omega$ and on the range space for the transform $L S P(\Omega) \ni \varphi \mapsto K_{\Omega(\varphi, m)}$ are described in Section 3. 
Without any further explanations we use the following symbols: $\mathbb{N}$ - the set of natural numbers; $\mathbb{Z}^{+}:=\mathbb{N} \cup\{0\} ; \mathbb{R}$ - the set of reals; $\mathbb{C}$ - the complex plane; $X^{m}$ - the $m$ th Cartesian power of the set $X$.

2. Admissible weights and weighted Bergman functions. Let $W(\Omega)$ be the set of all weights (of integration) on an open nonempty set $\Omega \subset \mathbb{C}^{n}$, i.e., of all Lebesgue measurable real-valued positive functions defined on $\Omega$. If $\mu \in W(\Omega)$ then $L^{2} H(\Omega, \mu)$ denotes the space of all $\mu$-square integrable holomorphic functions on $\Omega$. For any $z \in \Omega$ we define the evaluation functional $E_{z}$ on $L^{2} H(\Omega, \mu)$ as follows:

$$
E_{z} f:=f(z), \quad f \in L^{2} H(\Omega, \mu) .
$$

A weight $\mu$ on $\Omega$ is called admissible if $L^{2} H(\Omega, \mu)$ is a closed subspace of the Hilbert space $L^{2}(\Omega, \mu)$ of all $\mu$-square integrable functions on $\Omega$ and if for any $z \in \Omega$ the evaluation functional $E_{z}$ is continuous on $L^{2} H(\Omega, \mu)$. The set of all admissible weights on $\Omega$ will be denoted by $A W(\Omega)$ (see [11] and [12]). It is proved in [11] (see also [12]) that if $\mu \in W(\Omega)$ and $1 / \mu$ is locally integrable then $\mu$ is an admissible.

Let $\widetilde{U}(\Omega):=\left\{g \in L_{\mathbb{R}}^{\infty}(\Omega): \operatorname{essinf}_{z \in \Omega} g(z)>0\right\}$. We will consider $W(\Omega)$ as a differentiable (analytic) Banach manifold with the differential structure given by the atlas $\left\{\left(\widetilde{\Phi}_{\mu}^{-1}, \widetilde{\Phi}_{\mu}(\widetilde{U}(\Omega))\right), \mu \in W(\Omega)\right\}$, where for each $\mu \in W(\Omega)$ the map $\widetilde{\Phi}_{\mu}: \widetilde{U}(\Omega) \rightarrow W(\Omega)$ is defined by

$$
\left[\widetilde{\Phi}_{\mu}(g)\right](z):=g(z) \mu(z), \quad g \in \widetilde{U}(\Omega), z \in \Omega .
$$

It turns out that $A W(\Omega)$ is an open submanifold of $W(\Omega)$ (see [11]).

For $\mu \in A W(\Omega)$ the evaluation functional $E_{z}$ is uniquely represented by a function $e_{z, \mu} \in L^{2} H(\Omega, \mu)$ in the sense of the Riesz theorem. The function $K(\mu): \Omega \times \Omega \rightarrow \mathbb{C}$ given by

$$
[K(\mu)](z, w):=\overline{e_{z, \mu}(w)}, \quad z, w \in \Omega,
$$

is called the $\mu$-Bergman function of $\Omega$ (see [1], [11] or [12]). The following facts are basic for our study.

THEOREM 1. For any $\mu \in A W(\Omega)$ the function $K(\mu)$ has the following properties: in $w$;

(i) $[K(\mu)](z, w)=\overline{[K(\mu)](w, z)}, z, w \in \Omega$;

(ii) $[K(\mu)](z, w)$ is real-analytic, holomorphic in $z$ and antiholomorphic

(iii) if $P_{\mu}$ is the $\langle\cdot \mid \cdot\rangle_{\mu}$-orthogonal projection of $L^{2}(\Omega, \mu)$ onto $L^{2} H(\Omega, \mu)$ then for any $z \in \Omega$ and each $f \in L^{2}(\Omega, \mu)$

$$
\left[P_{\mu} f\right](z)=\int_{\Omega}[K(\mu)](z, w) f(w) \mu(w) d w^{2 n},
$$


i.e., $K(\mu)$ is the $\mu$-integral kernel of the operator $P_{\mu}$;

(iv) for any $z, w \in \Omega$

$$
\langle[K(\mu)](\cdot, z) \mid[K(\mu)](\cdot, w)\rangle_{\mu}=[K(\mu)](z, w),
$$

and

$$
\|[K(\mu)](\cdot, z)\|_{\mu}=\|[K(\mu)](z, \cdot)\|_{\mu}=[K(\mu)]^{1 / 2}(z, z) .
$$

Proof. For the proof of (i)-(iii) we refer to [12, Theorem 2.1]. The statement (iv) is an immediate consequence of (iii).

Let $H A(\Omega)$ be the real vector space of all complex-valued functions on $\Omega \times \Omega$ which are real-analytic, holomorphic with respect to the first $n$ variables and antiholomorphic with respect to the last $n$ variables. We endow $H A(\Omega)$ with the Fréchet space topology given by the family of seminorms $\left\{\|\cdot\|_{X}: X \subset \Omega, X\right.$ compact $\}$, where

$$
\|F\|_{X}:=\sup _{(z, w) \in X \times X}|F(z, w)|, \quad F \in H A(\Omega) .
$$

It now follows from Theorem 1 that the definition of a weighted Bergman function can be interpreted as the definition of a functional (nonlinear) transform $K: A W(\Omega) \rightarrow H A(\Omega)$. It is proved in [11, Theorem 5.1] that $K$ is analytic. Here we only need the formula for the $k$ th total derivative of the superposition $K \circ \widetilde{\Phi}_{\mu}$, where $\mu \in A W(\Omega)$ (see (1)). We have

$$
\left[D_{g}^{(k)} K(g \mu) h^{(k)}\right](z, w)=(-1)^{k} k !\left[K_{g, \mu}^{(k)} h^{(k)}\right](z, w),
$$

where

$$
\begin{aligned}
{[6) } & {\left[K_{g, \mu}^{(k)}\left(h_{1}, \ldots, h_{k}\right)\right](z, w):=\int_{\Omega}[K(g \mu)]\left(u_{1}, w\right) h_{1}\left(u_{1}\right) \mu\left(u_{1}\right) d u_{1}^{2 n} } \\
& \times \int_{\Omega}[K(g \mu)]\left(u_{2}, u_{1}\right) h_{2}\left(u_{2}\right) \mu\left(u_{2}\right) d u_{2}^{2 n} \\
& \cdots \int_{\Omega}[K(g \mu)]\left(u_{k}, u_{k-1}\right) h_{k}\left(u_{k}\right)[K(g \mu)]\left(z, u_{k}\right) \mu\left(u_{k}\right) d u_{k}^{2 n}, \\
g & \in \widetilde{U}(\Omega), h_{1}, \ldots, h_{k} \in L_{\mathbb{R}}^{\infty}(\Omega), z, w \in \Omega,
\end{aligned}
$$

and the integral on the right hand side is an iterated integral (see [11, Theorem 5.1, Corollary 5.1].

Proposition 1. Let $\mu \in A W(\Omega), g \in \widetilde{U}(\Omega)$ and let $e_{1}, \ldots, e_{k} \in L_{\mathbb{R}}^{\infty}(\Omega)$. Then for any $(z, w) \in \Omega \times \Omega$

$$
\begin{aligned}
\mid\left[K _ { g , \mu } ^ { ( k ) } \left(\left(e_{1} g\right), \ldots,\right.\right. & \left.\left.\left(e_{k} g\right)\right)\right](z, w) \mid \\
& \leq[K(g \mu)]^{1 / 2}(z, z)[K(g \mu)]^{1 / 2}(w, w)\left\|e_{1}\right\| \ldots\left\|e_{k}\right\| .
\end{aligned}
$$


Proof. Since

$$
\begin{aligned}
& {\left[K_{g, \mu}^{(k)}\left(\left(e_{1} g\right), \ldots,\left(e_{k} g\right)\right)\right](z, w)} \\
& =\overline{\int_{\Omega}[K(g \mu)]\left(w, u_{1}\right) e_{1}\left(u_{1}\right) g\left(u_{1}\right) \mu\left(u_{1}\right) d u_{1}^{2 n}} \\
& \times \overline{\int_{\Omega}[K(g \mu)]\left(u_{1}, u_{2}\right) e_{2}\left(u_{2}\right) g\left(u_{2}\right) \mu\left(u_{2}\right) d u_{2}^{2 n}} \\
& \cdots \int_{\Omega}[K(g \mu)]\left(u_{k-1}, u_{k}\right) e_{k}\left(u_{k}\right)[K(g \mu)]\left(u_{k}, z\right) g\left(u_{k}\right) \mu\left(u_{k}\right) d u_{k}^{2 n} \\
& =\overline{\left\{\left[P_{g \mu} \circ A\left(e_{1}\right)\right] \circ \ldots \circ\left[P_{g \mu} \circ A\left(e_{k}\right)\right][K(g \mu)](\cdot, z)\right\}(w)}, \quad z, w \in \Omega,
\end{aligned}
$$

where

$$
[A(e) f](z)=e(z) f(z), \quad e \in L_{\mathbb{R}}^{\infty}(\Omega), z \in \Omega, f \in L^{2}(\Omega, g \mu),
$$

we obtain (by Theorem 1 (iv))

$$
\begin{aligned}
& \left\|\left[K_{g, \mu}^{(k)}\left(\left(e_{1} g\right), \ldots,\left(e_{k} g\right)\right)\right](z, \cdot)\right\|_{g \mu} \\
& \quad \leq \prod_{i=1}^{k}\left\|A\left(e_{i}\right)\right\|_{g \mu}\|[K(g \mu)](\cdot, z)\|_{g \mu}=\prod_{i=1}^{k}\left\|e_{i}\right\|[K(g \mu)]^{1 / 2}(z, z) .
\end{aligned}
$$

Applying to the above inequality the formula for the norm of the evaluation functional $E_{w}$, i.e.,

$$
\left\|E_{w}\right\|_{g \mu}=\left\|e_{w, \mu}\right\|_{g \mu}=\|\overline{[K(g \mu)](w, \cdot)}\|_{g \mu}=[K(g \mu)]^{1 / 2}(w, w)
$$

we obtain (7).

Conollary 1. Under the assumptions of Proposition 1, if $X_{1}$ and $X_{2}$ are compact subsets of $\Omega$ and $e=h / g$, where $h \in L_{\mathbb{R}}^{\infty}(\Omega)$, then for each $(z, w) \in X_{1} \times X_{2}$

$$
\begin{aligned}
\left|\left[K_{g, \mu}^{(k)} h^{(k)}\right](z, w)\right| & =\left|\left[K_{g, \mu}^{(k)}\left(\frac{h}{g} g\right)^{(k)}\right](z, w)\right| \\
& \leq C_{x_{1}} C_{x_{2}}\|h / g\|^{k} \leq C_{x_{1}} C_{x_{2}}\left(\frac{\|h\|}{i(g)}\right)^{k},
\end{aligned}
$$

where $i(g)=\operatorname{essinf}_{z \in \Omega} g(z)$ and for any compact $X \subset \Omega$

$$
C_{X}:=\sup _{z \in X}[K(g \mu)]^{1 / 2}(z, z) .
$$

The classical Bergman space and the classical Bergman function for the set $\Omega \subset \mathbb{C}^{n}$ will be denoted by $L^{2} H(\Omega)$ and $K_{\Omega}$ respectively.

3. The Bergman function of the bounded Hartogs domain. From now on we will assume that $\Omega$ is bounded. 
Let $\operatorname{LSP}(\Omega)$ denote the set of all lower semicontinuous positive bounded functions on $\Omega$. It is clear that if $\varphi \in L S P(\Omega)$ then $1 / \varphi$ is locally integrable on $\Omega$. Consequently, $L S P(\Omega) \subset A W(\Omega)$.

Similarly to $A W(\Omega)$ the set $L S P(\Omega)$ can be endowed with the structure of a differentiable (analytic) Banach manifold. Namely, let $U(\Omega):=$ $\left\{g \in C_{\mathbb{R}}^{b}(\Omega): \inf _{z \in \Omega} g(z)>0\right\}$, where $C_{\mathbb{R}}^{b}(\Omega)$ is the Banach space of all real-valued bounded continuous functions on $\Omega$ (with the norm $\|g\|=$ $\left.\sup _{z \in \Omega}|g(z)|, g \in C_{\mathbb{R}}^{b}(\Omega)\right)$. Analogously to (1) we define $\Phi_{\varphi}: U(\Omega) \rightarrow$ $\operatorname{LSP}(\Omega)$ by

$$
\left[\Phi_{\varphi}(g)\right](z):=g(z) \varphi(z), \quad \varphi \in L S P(\Omega), g \in U(\Omega), z \in \Omega .
$$

The family $\left\{\left(\Phi_{\varphi}^{-1}, \Phi(U(\Omega)): \varphi \in L S P(\Omega)\right\}\right.$ is an atlas for a differentiable (analytic) Banach manifold structure on $L S P(\Omega)$. Since $L S P(\Omega) \subset A W(\Omega)$ and $C_{\mathbb{R}}^{b}(\Omega)$ is a closed subspace of $L_{\mathbb{R}}^{\infty}(\Omega)$ and for any $\varphi \in \operatorname{LSP}(\Omega)$

$$
\widetilde{\Phi}_{\varphi \mid U(\Omega)}=\Phi_{\varphi}
$$

we can apply the results of the previous section to the transform $K$ restricted to $L S P(\Omega)$. In particular, $K$ is analytic on $L S P(\Omega)$ and the formulas (5) and (6) for the total derivative as well as Proposition 1 and Corollary 1 hold in this case.

For any $\varphi \in L S P(\Omega)$ and any $m \in \mathbb{N}$ define

$$
\Omega(\varphi, m):=\left\{(z, \xi) \in \mathbb{C}^{n+m}: z \in \Omega, \xi \in \mathbb{C}^{m},|\xi|<\varphi(z)\right\} .
$$

This is the Hartogs domain defined by $m$ and $\varphi$ (and $\Omega$ ). Since $\varphi$ is lower semicontinuous and positive, $\Omega(\varphi, m)$ is nonempty and open in $\mathbb{C}^{n+m}$.

Let

$$
U H A(\Omega, m):=\bigcup_{\varphi \in L S P(\Omega)} H A(\Omega(\varphi, m)) .
$$

The main purpose of this paper is to investigate the map

$$
L S P(\Omega) \ni \varphi \mapsto \mathcal{B}_{m}(\varphi):=K_{\Omega(\varphi, m)} \in \operatorname{UHA}(\Omega, m),
$$

where $K_{\Omega(\varphi, m)}$ is the classical Bergman function of $\Omega(\varphi, m)$. We first endow the set $U H A(\Omega, m)$ with a suitable topological and differentiable structure. Namely, if $F \in U H A(\Omega, m)$ then we denote by $\varphi(F)$ an element of $\operatorname{LSP}(\Omega)$ such that $F \in H A(\Omega(\varphi(F), m))$. It is clear that $\varphi(F)$ is uniquely determined by $F$. For any $F_{0} \in U H A(\Omega, m)$, any compact set $Z \subset \Omega\left(\varphi\left(F_{0}\right), m\right)^{2}$ and any $\varepsilon>0$ we define the set $\mathcal{O}\left(F_{0}, Z, \varepsilon\right)$ as follows: $F \in \mathcal{O}\left(F_{0}, Z, \varepsilon\right)$ iff $Z \subset \Omega(\varphi(F), m)^{2}$ and for any $(z, w) \in Z$

$$
\left|F(z, w)-F_{0}(z, w)\right|<\varepsilon .
$$

It is easy to verify that the family $\left\{\mathcal{O}\left(F_{0}, Z, \varepsilon\right): F_{0} \in U H A(\Omega, m), Z \subset\right.$ $\Omega\left(\varphi\left(F_{0}\right), m\right)^{2}, Z$ compact, $\left.\varepsilon>0\right\}$ forms a basis of some topology $\tau$ on $U H A(\Omega, m)$ (see [9], XII, 1). 
If $Z$ is a compact subset of $\left(\Omega \times \mathbb{C}^{m}\right)^{2}$ then the set $\mathcal{O}(Z):=\{F \in$ $\left.U H A(\Omega, m): Z \subset \Omega(\varphi(F), m)^{2}\right\}$ is open in $U H A(\Omega, m)$. We define the map $\psi_{Z}: \mathcal{O}(Z) \mapsto C(Z)$ as the restriction

$$
\psi_{Z}(F)=F_{\mid Z}, \quad F \in \mathcal{O}(Z),
$$

where $C(Z)$ is the Banach space of all complex-valued continuous functions on $Z$ with the standard norm

$$
\|H\|_{Z}=\sup _{(z, w) \in Z}|H(z, w)|, \quad H \in C(Z) .
$$

Proposition 2. Let $M$ be a topological space. A map $\mathcal{F}: M \rightarrow$ $\operatorname{UHA}(\Omega, m)$ is continuous iff for any compact set $Z \subset\left(\Omega \times \mathbb{C}^{m}\right)^{2}$ the set $\mathcal{F}^{-1}[\mathcal{O}(Z)]$ is open in $M$ and the superposition

$$
\psi_{Z} \circ \mathcal{F}: \mathcal{F}^{-1}[\mathcal{O}(Z)] \rightarrow C(Z)
$$

is continuous.

We leave the proof to the reader.

The above considerations suggest the following definition of differentiability.

Definition 1. Let $M$ be a differentiable manifold (finite-dimensional or Banach). A map $\mathcal{F}: M \rightarrow U H A(\Omega, m)$ is said to be differentiable of class $C^{k}, k=0,1,2, \ldots, \infty$ or $\omega$, if for any compact set $Z \subset\left(\Omega \times \mathbb{C}^{m}\right)^{2}$ the set $\mathcal{F}^{-1}[\mathcal{O}(Z)]$ is open in $M$ and the superposition $\psi_{Z} \circ \mathcal{F}$ is differentiable of class $C^{k}$ on $\mathcal{F}^{-1}[\mathcal{O}(Z)]$.

It follows from Proposition 2 that any $C^{k} \operatorname{map} \mathcal{F}: M \rightarrow U H A(\Omega, m)$ is continuous.

Remark 1. In the present paper we do not consider the problem whether or not $U H A(\Omega, m)$ is a differentiable manifold.

In the remaining part of this section we describe the Rudin idea of representing the classical Bergman function of the Hartogs domain as the sum of an infinite series of weighted Bergman functions (see [6] or [7]).

Let $\varphi \in L S P(\Omega)$. If $f$ is a holomorphic function on $\Omega(\varphi, m)$ then

$$
f(z, \xi)=\sum_{|\alpha|=0}^{\infty} f_{\alpha}(z) \xi^{\alpha}, \quad z \in \Omega,(z, \xi) \in \Omega(\varphi, m),
$$

where $f_{\alpha}$ is holomorphic on $\Omega$ for any multiindex $\alpha \in\left(\mathbb{Z}^{+}\right)^{m}$ and the series converges uniformly on any compact subset of $\Omega(\varphi, m)$. This series is called the Hartogs series of $f$ (see [5] or [14]).

Proposition 3. Let $\varphi \in \operatorname{LSP}(\Omega)$ and $m \in \mathbb{N}$.

(i) A function $f$ holomorphic on $\Omega(\varphi, m)$ is square integrable iff for any multiindex $\alpha \in\left(\mathbb{Z}^{+}\right)^{m}$ the $\alpha$-coefficient $f_{\alpha}$ of $f$ in the Hartogs series is in 
$L^{2} H\left(\Omega, c_{\alpha} \varphi^{2|\alpha|+2 m}\right)$ and

$$
\sum_{|\alpha|=0}^{\infty}\left\|f_{\alpha}\right\|_{c_{\alpha} \varphi^{2|\alpha|+2 m}}^{2}<\infty
$$

where $c_{\alpha}>0$ is a suitable constant. Moreover,

$$
\|f\|_{L^{2}}^{2}=\sum_{|\alpha|=0}^{\infty}\left\|f_{\alpha}\right\|_{c_{\alpha} \varphi^{2|\alpha|+2 m}}^{2} .
$$

(ii) If $K_{\Omega(\varphi, m)}$ is the Bergman function of $\Omega(\varphi, m)$ then for any $(z, \xi)$, $(w, \eta) \in \Omega(\varphi, m)$

$$
K_{\Omega(\varphi, m)}((z, \xi),(w, \eta))=\sum_{|\alpha|=0}^{\infty} \xi^{\alpha} K_{\alpha}(z, w) \bar{\eta}^{\alpha},
$$

where $K_{\alpha}=K\left(c_{\alpha} \varphi^{2|\alpha|+2 m}\right)$ is the $\left(c_{\alpha} \varphi^{2|\alpha|+2 m}\right)$-Bergman function on $\Omega \times \Omega$. The series on the right hand side converges uniformly on any compact subset of $\Omega(\varphi, m) \times \Omega(\varphi, m)$.

For the proof we refer to [7].

Remark 2. If $\varphi \in L S P(\Omega)$ then for any $c>0$ and any $p \in \mathbb{N}$ the function $c \varphi^{p}$ is in $L S P(\Omega)$ and therefore $c \varphi^{p} \in A W(\Omega)$.

4. Smoothness of the map $L S P(\Omega) \ni \varphi \mapsto \mathcal{B}_{m}(\varphi):=K_{\Omega(\varphi, m)} \in$ $U H A(\Omega, m)$. Fix $\varphi \in L S P(\Omega)$ and consider the superposition $\mathcal{B}_{m} \circ \Phi_{\varphi}$ (see $(9)$ ), i.e., the transform

$$
U(\Omega) \ni g \mapsto \mathcal{B}_{m}(g \varphi) \in U H A(\Omega, m) .
$$

For any $\alpha \in\left(\mathbb{Z}^{+}\right)^{m}$ define $H_{\alpha}(g):=K\left(c_{\alpha}(g \varphi)^{2|\alpha|+2 m}\right)$. Then by Proposition 3

$$
\begin{array}{r}
{\left[\mathcal{B}_{m}(g \varphi)\right]((z, \xi),(w, \eta))=\sum_{|\alpha|=0}^{\infty} \xi^{\alpha}\left[H_{\alpha}(g)\right](z, w) \bar{\eta}^{\alpha}} \\
(z, \xi),(w, \eta) \in \Omega(g \varphi, m) .
\end{array}
$$

It follows from (5) and (6) that

$$
\begin{aligned}
& {[19) \quad\left[H_{\alpha}(g) h\right](z, w) } \\
&=\left\{\left[\left(\left.D_{f} K\left(c_{\alpha} \varphi^{2|\alpha|+2 m} f\right)\right|_{\left.\left.\left.f=g^{2|\alpha|+2 m}\right) D_{g} g^{2|\alpha|+2 m}\right] h\right\}(z, w)}\right.\right.\right. \\
&=-(2|\alpha|+2 m) \int_{\Omega}\left[H_{\alpha}(g)\right](u, w) \frac{h(u)}{g(u)} \\
& \times\left[H_{\alpha}(g)\right](z, u)(g(u) \varphi(u))^{2|\alpha|+2 m} d u^{2 n}, \\
& \alpha \in\left(\mathbb{Z}^{+}\right)^{m}, h \in \mathbb{C}_{\mathbb{R}}^{b}(\Omega),(z, w) \in \Omega \times \Omega .
\end{aligned}
$$


We want to show that the transform (17) is differentiable and

$$
\begin{array}{r}
{\left[D_{g} \mathcal{B}_{m}(g \varphi) h\right]((z, \xi),(w, \eta))=\sum_{|\alpha|=0}^{\infty} \xi^{\alpha}\left[D H_{\alpha}(g) h\right](z, w) \bar{\eta}^{\alpha},} \\
h \in C_{\mathbb{R}}^{b}(\Omega),(z, \xi),(w, \eta) \in \Omega(g \varphi, m) .
\end{array}
$$

Proposition 4. The series on the right hand side of (20) converges uniformly on any compact subset of $\Omega(g \varphi, m)^{2}$.

Pr o of. Using Proposition 1 and the Schwarz inequality in the space $l^{2}$ we get

$$
\begin{aligned}
& \sum_{|\alpha|=k}^{\infty}(2|\alpha|+2 m) \mid \xi^{\alpha} \bar{\eta}^{\alpha} \int_{\Omega}\left[H_{\alpha}(g)\right](u, w) \frac{h(u)}{g(u)}\left[H_{\alpha}(g)\right](z, u) \\
& \times(g(u) \varphi(u))^{2|\alpha|+2 m} d u^{2 n} \mid \\
& \leq \sum_{|\alpha|=k}^{\infty} 2(|\alpha|+m)\left|\xi^{\alpha}\right|\left[H_{\alpha}(g)\right]^{1 / 2}(z, z)\left|\eta^{\alpha}\right|\left[H_{\alpha}(g)\right]^{1 / 2}(w, w)\|h / g\| \\
& \leq\left(\sum_{|\alpha|=k}^{\infty} 2(|\alpha|+m)\left|\xi^{\alpha}\right|^{2}\left[H_{\alpha}(g)\right](z, z)\right)^{1 / 2} \\
& \times\left(\sum_{|\alpha|=k}^{\infty} 2(|\alpha|+m)\left|\eta^{\alpha}\right|\left[H_{\alpha}(g)\right](w, w)\right)^{1 / 2}\|h / g\|, \\
&(z, \xi),(w, \eta) \in \Omega(g \varphi, m), \quad k \in \mathbb{N} .
\end{aligned}
$$

Note that by (18)

$$
\begin{aligned}
& \sum_{|\alpha|=0}^{\infty} 2(|\alpha|+m)\left|\xi^{\alpha}\right|^{2}\left[H_{\alpha}(g)\right](z, z) \\
& =\left.2 \sum_{j=1}^{m} \xi_{j} \frac{\partial\left[\mathcal{B}_{m}(g \varphi)\right]((z, \xi),(w, \eta))}{\partial \xi_{j}}\right|_{\substack{z=w \\
\xi=\eta}} \\
& +2 m\left[\mathcal{B}_{m}(g \varphi)\right]((z, \xi),(z, \xi))<\infty
\end{aligned}
$$

Analogously $\sum_{|\alpha|=0}^{\infty} 2(|\alpha|+m)\left|\xi^{\alpha}\right|^{2}\left[H_{\alpha}(g)\right](w, w)<\infty$ and consequently, by (19), the considered series converges for any $(z, \xi),(w, \eta) \in \Omega(g \varphi, m)$. It now follows from the Dini theorem that the series in (22) converges uniformly on any compact subset of $\Omega(g \varphi, m)^{2}$. Hence using once more (19) and the inequality $(21)$ for $k \rightarrow \infty$ we obtain the uniform convergence of the series in (20) on compact subsets of $\Omega(g \varphi, m)^{2}$. 
Lemma 1. Let $\varphi \in \operatorname{LSP}(\Omega), g \in U(\Omega)$ and let $h \in C_{\mathbb{R}}^{b}(\Omega)$ be such that $\|h\|<i(g) / 2$, where $i(g):=\inf _{z \in \Omega} g(z)$. Then for any $z \in \Omega$

$$
\begin{aligned}
{\left[H_{\alpha}(g)\right](z, z) e^{-4(|\alpha|+m)\|h\| / i(g)} } & \leq\left[H_{\alpha}(g+h)\right](z, z) \\
& \leq\left[H_{\alpha}(g)\right](z, z) e^{4(|\alpha|+m)\|h\| / i(g)} .
\end{aligned}
$$

Proof. Fix $z \in \Omega$ and $\alpha \in\left(\mathbb{Z}^{+}\right)^{m}$. Let $x(t):=\left[H_{\alpha}(g+t h)\right](z, z)$, $t \in[0,1]$. Then $x$ is differentiable and by (19)

$$
\begin{aligned}
\frac{d x(t)}{d t}= & {\left[D H_{\alpha}(g+t h) h\right](z, z) } \\
= & -2(|\alpha|+m) \int_{\Omega}\left[H_{\alpha}(g+t h)\right](u, z) \frac{h(u)}{g(u)+t h(u)} \\
& \times\left[H_{\alpha}(g+t h)\right](z, u)(g(u)+t h(u))^{2(|\alpha|+m)} d u^{2 m} .
\end{aligned}
$$

Applying Proposition 1 to the above equality we obtain

$$
\begin{aligned}
\left|\frac{d x(t)}{d t}\right| & \leq 2(|\alpha|+m)\left[H_{\alpha}(g+t h)\right](z, z) \frac{\|h\|}{i(g+t h)} \\
& \leq 4(|\alpha|+m) \frac{\|h\|}{i(g)} x(t),
\end{aligned}
$$

where we have used the inequality $i(g+t h)>i(g) / 2$, which follows from the assumptions of the lemma. Since $\Omega, h$ and $\varphi$ are bounded we see that for any $w \in \Omega$ there exists $f \in L^{2} H\left(\Omega, c_{\alpha}((g+t h) \varphi)^{2|\alpha|+2 m}\right)$ such that $f(w) \neq 0$. For example, we can take $f=\chi_{\Omega}$, the characteristic function of $\Omega$. Then, by [10], $x(t)=\left[H_{\alpha}(g+t h)\right](z, z)>0$ for each $t \in[0,1]$. Dividing now all members of $(24)$ by $x(t)$ and integrating over $[0, s], s \in[0,1]$, we get

$$
-4(|\alpha|+m) \frac{\|h\|}{i(g)} s \leq \ln \frac{x(s)}{x(0)} \leq 4(|\alpha|+m) \frac{\|h\|}{i(g)} s .
$$

Putting $s=1$ and passing to exponential functions we obtain (23).

Now we are in a position to prove the main result of the present paper.

Theorem 2. For each $m \in \mathbb{N}$ the map

$$
L S P(\Omega) \ni \varphi \mapsto \mathcal{B}_{m}(\varphi)=K_{\Omega(\varphi, m)} \in \operatorname{UHA}(\Omega, m)
$$

is smooth, i.e., it is of class $C^{\infty}$.

Proof. It follows from the definition of the differential structures on $\operatorname{LSP}(\Omega)$ and $\operatorname{UHA}(\Omega, m)$ that we should show the smoothness of the maps $(25) \quad\left(\mathcal{B}_{m} \circ \Phi_{\varphi}\right)^{-1}[\mathcal{O}(Z)] \ni g \mapsto\left(\Psi_{z} \circ \mathcal{B}_{m} \circ \Phi_{\varphi}\right)(g)=\mathcal{B}_{m}(g \varphi)_{\mid Z} \in C(Z)$, where $\varphi \in \operatorname{LSP}(\Omega)$ and $Z$ is an arbitrary compact subset of $\left(\Omega \times \mathbb{C}^{m}\right)^{2}$. It is clear that $\left(\mathcal{B}_{m} \circ \Phi_{\varphi}\right)^{-1}[\mathcal{O}(Z)]=\left\{g \in U(\Omega): Z \subset(\Omega(g \varphi, m))^{2}\right\}$ is open in $U(\Omega)$, which implies that $\mathcal{B}_{m}^{-1}[\mathcal{O}(Z)]$ is open in $L S P(\Omega)$. 
To avoid tedious considerations we will only prove rigorously the fact that $\mathcal{B}_{m}$ is of class $C^{1}$. Moreover, note that any compact subset $Z$ of $\left(\Omega \times \mathbb{C}^{m}\right)^{2}$ can be covered by a finite sum $\bigcup_{i, j=1}^{p} Z_{i, j}$ of compact sets which have the form

$$
Z_{i, j}=\left(Y_{i} \times \overline{B\left(0, R_{i}\right)}\right) \times\left(Y_{j} \times \overline{B\left(0, R_{j}\right)}\right),
$$

where $Y_{i} \subset \Omega$ is compact and $\overline{B\left(0, R_{i}\right)}=\left\{\xi \in \mathbb{C}^{m}:|\xi| \leq R_{i}\right\}$ is a closed ball in $\mathbb{C}^{m}$ for $i=1, \ldots, p$. Moreover, if $Z \subset \Omega(\Psi, m)^{2}$ for some $\Psi \in L S P(\Omega)$ one can choose $Y_{j}$ and $R_{j}$ in such a way that $Z_{i, j} \subset \Omega(\Psi, m)^{2}$ for $i, j=1, \ldots, p$. Hence it is enough to assume that $Z=Z_{1,2}$ (see (26)).

Consider the second derivative of $H_{\alpha}$, where $\alpha \in\left(\mathbb{Z}^{+}\right)^{m}$. By direct calculations (using (5) and the chain rule) we obtain

$$
\begin{aligned}
& {\left[D^{(2)} H_{\alpha}(g)\left(h_{1}, h_{2}\right)\right](z, w) } \\
= & (2|\alpha|+2 m)^{2}\left\{\int_{\Omega}[H(g)]\left(u_{1}, w\right) \frac{h_{1}\left(u_{1}\right)}{g\left(u_{1}\right)} c_{\alpha}\left[g\left(u_{1}\right) \varphi\left(u_{1}\right)\right]^{2|\alpha|+2 m} d u_{1}^{2 n}\right. \\
\times & \int_{\Omega}\left[H_{\alpha}(g)\right]\left(u_{2}, u_{1}\right) \frac{h_{2}\left(u_{2}\right)}{g\left(u_{2}\right)}\left[H_{\alpha}(g)\right]\left(z, u_{2}\right) c_{\alpha}\left[g\left(u_{2}\right) \varphi\left(u_{2}\right)\right]^{2|\alpha|+2 m} d u_{2}^{2 n} \\
+ & \int_{\Omega}\left[H_{\alpha}(g)\right]\left(u_{1}, w\right) \frac{h_{2}\left(u_{1}\right)}{g\left(u_{1}\right)} c_{\alpha}\left[g\left(u_{1}\right) \varphi\left(u_{1}\right)\right]^{2|\alpha|+2 m} d u_{1}^{2 n} \\
\times & \left.\left.\int_{\Omega}\left[H_{\alpha}(g)\right]\left(u_{2}, u_{1}\right) \frac{h_{1}\left(u_{2}\right)}{g\left(u_{2}\right)}\left[H_{\alpha}(g)\right]\left(z, u_{2}\right) c_{\alpha}\left[g\left(u_{2}\right) \varphi\left(u_{2}\right)\right]^{2|\alpha|+2 m}\right] d u_{2}^{2 n}\right\} \\
- & (2|\alpha|+2 m)(2|\alpha|+2 m-1) \quad \int_{\Omega}\left[H_{\alpha}(g)\right](u, w) \frac{h_{1}(u) h_{2}(u)}{g(u)^{2}}\left[H_{\alpha}(g)\right](z, u) \\
\times & c_{\alpha}[g(u) \varphi(u)]^{2|\alpha|+2 m} d u^{2 n}, \quad g \in U(\Omega), h_{1}, h_{2} \in C_{\mathbb{R}}^{b}(\Omega), z, w \in \Omega .
\end{aligned}
$$

Hence, by Proposition 1,

$$
\begin{aligned}
& \left|\left[D^{(2)} H_{\alpha}(g)\left(h_{1}, h_{2}\right)\right](z, w)\right| \\
& \leq\left[2(2|\alpha|+2 m)^{2}+(2|\alpha|+2 m)(2|\alpha|+2 m-1)\right] \\
& \quad \times\left[H_{\alpha}(g)\right]^{1 / 2}(z, z)\left[H_{\alpha}(g)\right]^{1 / 2}(w, w)\left\|h_{1} / g\right\| \cdot\left\|h_{2} / g\right\| \\
& \leq a(\alpha, m)\left[H_{\alpha}(g)\right]^{1 / 2}(z, z)\left[H_{\alpha}(g)\right]^{1 / 2}(w, w) \frac{\left\|h_{1}\right\| \cdot\left\|h_{2}\right\|}{i(g)^{2}}, \\
& g \in U(\Omega), h_{1}, h_{2} \in C_{\mathbb{R}}^{b}(\Omega), z, w \in \Omega, \alpha \in\left(\mathbb{Z}^{+}\right)^{m},
\end{aligned}
$$

where

$$
a(\alpha, m):=12|\alpha|^{2}+(24 m-2)|\alpha|+12 m^{2}-2 m .
$$

Let

$(28) \quad\left[\mathcal{D}^{(2)} \mathcal{B}_{m}(g \varphi)\left(h_{1}, h_{2}\right)\right]((z, \xi),(w, \eta))$ 


$$
:=\sum_{|\alpha|=0}^{\infty} \xi^{\alpha}\left[D^{(2)} H_{\alpha}(g)\left(h_{1}, h_{2}\right)\right](z, w) \bar{\eta}^{\alpha}, \quad(z, \xi),(w, \eta) \in \Omega(g \varphi, m) .
$$

Using (27) and the Schwarz inequality we get

$$
\begin{aligned}
& \sum_{|\alpha|=k}^{\infty}\left|\xi^{\alpha}\left[D^{(2)} H_{\alpha}(g)\left(h_{1}, h_{2}\right)\right](z, w) \bar{\eta}^{\alpha}\right| \\
\leq & \left(\sum_{|\alpha|=k}^{\infty} a(\alpha, m)\left[H_{\alpha}(g)\right](z, z)\left|\xi^{\alpha}\right|^{2}\right)^{1 / 2} \\
& \times\left(\sum_{|\alpha|=k}^{\infty} a(\alpha, m)\left[H_{\alpha}(g)\right](w, w)\left|\eta^{\alpha}\right|^{2}\right)^{1 / 2} \frac{\left\|h_{1}\right\| \cdot\left\|h_{2}\right\|}{i(g)^{2}}, \\
& (z, \xi),(w, \eta) \in \Omega(g \varphi, m), k \in \mathbb{N} .
\end{aligned}
$$

Note that for a given $(z, \xi) \in \Omega(g \varphi, m)$,

$$
\begin{aligned}
\sum_{|\alpha|=0}^{\infty}|\alpha|^{2} & {\left[H_{\alpha}(g)\right](z, z)\left|\xi^{\alpha}\right|^{2} } \\
= & \left.\sum_{i, j=1}^{m} \xi_{i} \bar{\eta}_{j} \frac{\partial^{2}\left[\mathcal{B}_{m}(g \varphi)\right]((z, \xi),(w, \eta))}{\partial \xi_{i} \partial \bar{\eta}_{j}}\right|_{\substack{z=w \\
\xi=\eta}}<\infty .
\end{aligned}
$$

Similarly

$$
\begin{aligned}
& \sum_{|\alpha|=0}^{\infty}|\alpha|\left[H_{\alpha}(g)\right](z, z)\left|\xi^{\alpha}\right|^{2} \\
& \quad=\left.\sum_{j=1}^{m} \xi_{j} \frac{\partial\left[\mathcal{B}_{m}(g \varphi)\right]((z, \xi),(w, \eta))}{\partial \xi_{j}}\right|_{\substack{z=w \\
\xi=\eta}}<\infty,
\end{aligned}
$$

and

(32) $\quad \sum_{|\alpha|=0}^{\infty} m\left[H_{\alpha}(g)\right](z, z)\left|\xi^{\alpha}\right|^{2}=m\left[\mathcal{B}_{m}(g \varphi)\right]((z, \xi),(w, \eta))<\infty$.

This means that the series on the right hand side of (28) converges absolutely on $\Omega(g \varphi, m)^{2}$. Analogously to the proof of Proposition 4 one can show that it converges uniformly on any compact subset of $\Omega(g \varphi, m)^{2}$.

By (29) and (23), if $\left\|h_{1}\right\|,\left\|h_{2}\right\|<i(g) / 2$ then

(33) $\sum_{|\alpha|=0}^{\infty}\left|\xi^{\alpha}\left[D^{(2)} H_{\alpha}\left(g+t h_{2}\right)\left(h_{1}, h_{2}\right)\right](z, w) \bar{\eta}^{\alpha}\right|$ 


$$
\begin{aligned}
\leq & \left(\sum_{|\alpha|=0}^{\infty} a(\alpha, m)\left[H_{\alpha}(g)\right](z, z) e^{4(|\alpha|+m)\left\|h_{2}\right\| / i(g)}\left|\xi^{\alpha}\right|^{2}\right)^{1 / 2} \\
& \times\left(\sum_{|\alpha|=0}^{\infty} a(\alpha, m)\left[H_{\alpha}(g)\right](w, w) e^{4(|\alpha|+m)\left\|h_{2}\right\| / i(g)}\left|\eta^{\alpha}\right|^{2}\right)^{1 / 2} \\
& \times \frac{4\left\|h_{1}\right\| \cdot\left\|h_{2}\right\|}{i(g)^{2}}, \quad t \in[0,1] .
\end{aligned}
$$

Let $Z=Z_{1,2}$ (see (26)) be a compact subset of $\Omega(g \varphi, m)^{2}$. There exists $r>0$ such that for any $(z, \xi)=\left(z ; \xi_{1}, \ldots, \xi_{m}\right) \in Y_{i} \times \overline{B\left(0, R_{i}\right)}$

$$
\left(z ;\left|\xi_{1}\right|+r,\left|\xi_{2}\right|+r, \ldots,\left|\xi_{m}\right|+r\right) \in \Omega(g \varphi, m), \quad i=1,2 .
$$

Write $\xi \dot{+} r:=\left(\left|\xi_{1}\right|+r, \ldots,\left|\xi_{m}\right|+r\right)$ for $\xi=\left(\xi_{1}, \ldots, \xi_{m}\right) \in \mathbb{C}^{m}$ and $Z^{r}:=$ $\left.\{(z, \xi \dot{+} r),(w, \eta \dot{+} r)) \in\left(\mathbb{C}^{n} \times \mathbb{C}^{m}\right)^{2}:((z, \xi),(w, \eta)) \in Z\right\}$. It is clear that $Z^{r}$ is a compact subset of $\Omega(g \varphi, m)^{2}$. Let $\sigma>0$ be such that

$$
\sigma \leq\left(1-\frac{m}{m+1}\right) \frac{i(g)}{2} \ln \left(1+\frac{r}{R_{i}}\right), \quad i=1,2 .
$$

Then for any $\alpha \in\left(\mathbb{Z}^{+}\right)^{m}$

$$
\sigma \leq\left(1-\frac{m}{|\alpha|+m}\right) \frac{i(g)}{2} \ln \left(1+\frac{r}{R_{i}}\right)
$$

which implies

$$
e^{2(|\alpha|+m) \sigma / i(g)} \leq\left(1+r / R_{i}\right)^{|\alpha|}, \quad i=1,2 .
$$

Since for any $(z, \xi) \in Y_{i} \times \overline{B\left(0, R_{i}\right)}$

$$
\left(1+\frac{r}{R_{i}}\right)^{|\alpha|} \leq\left(\frac{\left|\xi_{1}\right|+r}{\left|\xi_{1}\right|}\right)^{\alpha_{1}} \ldots\left(\frac{\left|\xi_{m}\right|+r}{\left|\xi_{m}\right|}\right)^{\alpha_{m}}, \quad \alpha=\left(\alpha_{1}, \ldots, \alpha_{m}\right)
$$

we obtain

$$
e^{4(|\alpha|+m) \sigma / i(g)}\left|\xi^{\alpha}\right|^{2} \leq\left(\left|\xi_{1}\right|+r\right)^{2 \alpha_{1}} \ldots\left(\left|\xi_{m}\right|+r\right)^{2 \alpha_{m}} .
$$

Hence, for $\left\|h_{2}\right\|<\sigma$,

$$
\text { (34) } \begin{aligned}
& \sum_{|\alpha|=0}^{\infty} a(\alpha, m)\left[H_{\alpha}(g)\right](z, z) e^{4(|\alpha|+m)\left\|h_{2}\right\| / i(g)}\left|\xi^{\alpha}\right|^{2} \\
& \leq \sum_{|\alpha|=0}^{\infty} a(\alpha, m)\left[H_{\alpha}(g)\right](z, z)\left(\left|\xi_{1}\right|+r\right)^{2 \alpha_{1}} \cdots\left(\left|\xi_{m}\right|+r\right)^{2 \alpha_{m}} \leq C_{r}^{i}, \\
&(z, \xi) \in X_{i}:=Y_{i} \times \overline{B\left(0, R_{i}\right)}, \quad i=1,2,
\end{aligned}
$$


where

$$
\begin{aligned}
C_{r}^{i}:= & \sup _{(v, \kappa) \in X_{i}^{r}}\left[\left.12 \sum_{k, j=1}^{m} \xi_{k} \bar{\eta}_{j} \frac{\partial^{2}\left[\mathcal{B}_{m}(g \varphi)\right]((z, \xi),(w, \eta))}{\partial \xi_{k} \partial \bar{\eta}_{j}}\right|_{\substack{z=w=v \\
\xi=\eta=\kappa}}\right. \\
& +\left.(24 m-2) \sum_{j=1}^{m} \xi_{j} \frac{\partial\left[\mathcal{B}_{m}(g \varphi)\right]((z, \xi),(w, \eta))}{\partial \xi_{j}}\right|_{\substack{z=w=v \\
\xi=\eta=\kappa}} \\
& \left.+\left(12 m^{2}-2 m\right)\left[\mathcal{B}_{m}(g \varphi)\right]((v, \kappa),(v, \kappa))\right]
\end{aligned}
$$

and $X_{i}^{r}=\left\{(z, \xi \dot{+} r) \in \Omega(g \varphi, m):(z, \xi) \in X_{i}\right\}$ for $i=1,2$ (see (30)-(32) and $(27))$. Consequently, the series on the left hand side of (33) converges absolutely on $[0,1] \times Z$. Using arguments similar to the proof of Proposition 4 we conclude that this series converges uniformly on $[0,1] \times Z$.

Now let $\delta>0$ be such that $\delta<\min \{i(g) / 2, \sigma\}$ and for any $h \in C_{\mathbb{R}}^{b}(\Omega)$ the condition $\|h\|<\delta$ implies $Z, Z^{r} \subset \Omega((g+h) \varphi, m)^{2}$. If $\|h\|<\delta$ and $((z, \xi),(w, \eta)) \in Z$ then by the Taylor formula (see [8])

$$
\begin{aligned}
& \mid\left[\mathcal{B}_{m}((g+h) \varphi)\right]((z, \xi),(w, \eta)) \\
- & \left.-\sum_{|\alpha|=0}^{\infty} \xi_{m}(g \varphi)\right]((z, \xi),(w, \eta)) \\
\leq & \sum_{|\alpha|=0}^{\infty}\left|\left[H_{\alpha}(g+h)\right](z, w)-\left[H_{\alpha}(g)\right](z, w)-\left[D H_{\alpha}(g) h\right](z, w)\right| \cdot\left|\xi^{\alpha} \bar{\eta}^{\alpha}\right| \\
\leq & \sum_{|\alpha|=0}^{\infty} \int_{0}^{1}(1-t)\left|\left[D^{(2)} H_{\alpha}(g+t h) h^{(2)}\right](z, w) \xi^{\alpha} \bar{\eta}^{\alpha}\right| d t \leq 4 C_{r}^{1} C_{r}^{2} \frac{\|h\|^{2}}{i(g)^{2}}
\end{aligned}
$$

(see (33)), which means that (20) is true.

In order to prove the continuity of the map

$$
U(\Omega) \ni g \mapsto D_{g}\left[\mathcal{B}_{m}(g \varphi)\right]_{\left.\right|_{Z}} \in L\left(C_{\mathbb{R}}^{b}(\Omega), C(Z)\right)
$$

note that

$$
\begin{aligned}
& \left|\left[D_{g} \mathcal{B}_{m}\left(\left(g+h_{2}\right) \varphi\right) h_{1}\right]((z, \xi),(w, \eta))-\left[D_{g} \mathcal{B}_{m}(g \varphi) h_{1}\right]((z, \xi),(w, \eta))\right| \\
& \leq \sum_{|\alpha|=0}^{\infty}\left|\xi^{\alpha}\left(\left[D H_{\alpha}\left(g+h_{2}\right) h_{1}\right](z, w)-\left[D H_{\alpha}(g) h_{1}\right](z, w)\right) \bar{\eta}^{\alpha}\right| \\
& =\sum_{|\alpha|=0}^{\infty}\left|\xi^{\alpha} \bar{\eta}^{\alpha} \int_{0}^{1}\left[D^{(2)} H_{\alpha}\left(g+t h_{2}\right)\left(h_{1}, h_{2}\right)\right](z, w) d t\right| \leq 4 C_{r}^{1} C_{r}^{2} \frac{\left\|h_{1}\right\| \cdot\left\|h_{2}\right\|}{i(g)^{2}}
\end{aligned}
$$


(see (33) and (34)). Passing to the operator norms we get

$$
\left\|D_{g} \mathcal{B}_{m}\left(\left(g+h_{2}\right) \varphi\right)-D_{g} \mathcal{B}_{m}(g \varphi)\right\| \leq 4 C_{r}^{1} C_{r}^{2}\left\|h_{2}\right\| / i(g)^{2},
$$

which means that $D_{g} \mathcal{B}_{m}(g \varphi)$ is a continuous function of $g$. Using analogous methods and applying induction one can prove that $\mathcal{B}_{m}$ is differentiable of any order. We leave the details to the reader.

Remark 3. The question whether or not $\mathcal{B}_{m}$ is an analytic map will be considered in another paper.

Acknowledgments. I express my thanks to E. Ligocka for her inspiration and fruitful conversations on the topics of this study. I also wish to thank my wife for her help.

\section{References}

[1] J. Burbea and P. Masani, Banach and Hilbert Spaces of Vector-Valued Functions, Res. Notes Math. 90, Pitman, Boston 1984.

[2] F. Forelli and W. Rudin, Projections on spaces of holomorphic functions in balls, Indiana Univ. Math. J. 24 (1974), 593-602.

[3] R. E. Greene and S. G. Krantz, Stability of the Bergman kernel and curvature properties of bounded domains, in: Recent Developments in Several Complex Variables, J. Fornaess (ed.), Ann. of Math. Stud. 100, Princeton Univ. Press, Princeton, N.J., 1981.

[4] -, -, Deformation of complex structures, estimates for the $\bar{\partial}$ equation, and stability of the Bergman kernel, Adv. in Math. 43 (1982), 1-86.

[5] S. G. Krantz, Function Theory of Several Complex Variables, Interscience-Wiley, New York 1982.

[6] E. Ligocka, The regularity of the weighted Bergman projection, in: Seminar of Deformation Theory 1982/84, Lecture Notes in Math. 1165, Springer, 1985, 197203.

[7] _- On the Forelli-Rudin construction and weighted Bergman projections, Studia Math. 94 (1989), 257-272.

[8] K. Maurin, Analysis, Part 1, Elements, PWN-Reidel, Warszawa-Dordrecht 1976.

[9] -, Analysis, Part 2, Integration, Distributions, Holomorphic Functions, Tensor and Harmonic Analysis, PWN-Reidel, Warszawa-Dordrecht 1980.

[10] T. Mazur, On the complex manifolds of Bergman type, to appear.

[11] Z. Pasternak-Winiarski, On the dependence of the reproducing kernel on the weight of integration, J. Funct. Anal. 94 (1990), 110-134.

[12] —, On weights which admit the reproducing kernel of Bergman type, Internat. J. Math. and Math. Sci., to appear.

[13] W. Rudin, Function Theory in the Unit Ball in $\mathbb{C}^{n}$, Springer, Berlin 1980.

[14] B. V. Shabat, Introduction to Complex Analysis, 3rd ed., Nauka, Moscow 1985 (in Russian).

INSTITUTE OF MATHEMATICS

WARSAW UNIVERSITY OF TECHNOLOGY

PL POLITECHNIKI 1

00-661 WARSZAWA, POLAND

Reçu par la Rédaction le 14.9.1990 